\title{
EDITORIAL
}

\section{The end of the beginning?}

\author{
Thirty-five years have passed since a 'war on cancer' was declared, but despite billions of \\ dollars of investment, decisive victory is still distant. However, with limitations in standard \\ development strategies for anticancer drugs being increasingly addressed, the opportunity \\ to start winning more battles is growing.
}

\$200 billion, even to those familiar with the scale of the costs of drug discovery and development, is a massive investment. But by some estimates ${ }^{1}$, at least this amount has been spent on basic and clinical cancer research in the United States alone since Richard Nixon called for "an intensive campaign to find a cure for cancer" in 1971.

There is no doubt that this investment has catalysed huge advances in our understanding of the biology of cancer. But the pace of translation of this knowledge into therapies that might be considered to be a cure for cancer has been frustratingly slow. With a few exceptions - such as the successful long-term treatment of chronic myeloid leukaemia with the pioneering targeted drug imatinib - the proportion of patients with most cancers that survive 5 years or more after their diagnosis has not improved dramatically since the 1970s. Indeed, even the latest generation of targeted therapies have at best achieved additional survival benefits measured in months in clinical trials for the cancers that claim the majority of lives each year, such as advanced lung cancer and colon cancer.

This lack of therapeutic progress has prompted much debate on where the war on cancer has gone wrong, as highlighted by an article in Fortune two years ago entitled "Why we're losing the war on cancer -and how to win it"1. Among the key culprits identified in this article were standard mouse models of cancer, which are clearly far from optimal - around $90 \%$ of potential drugs that show promise in these models subsequently fail in clinical trials. More candidate anticancer drugs fail in Phase II trials - when efficacy in humans is generally first assessed - than in any other major therapeutic area ${ }^{2}$, emphasizing how poorly efficacy in current preclinical models predicts that in humans.

Why are the standard preclinical models of cancer so bad at predicting therapeutic outcomes in humans? Why are they still routinely used for evaluating anticancer drug candidates? And, most importantly, is anything being done to tackle the problem? All three questions are considered in a review on page 741 of this issue.
The first question is the simplest to answer: the standard mouse models are far from faithful replicas of the intricacies of human cancer. Typically, cultured cells derived from human tumours are injected into immunodeficient mice, and compounds are assessed for their effects on the resultant tumours. Such xenograft systems therefore model cancer more as if it is simply a disease of homogeneous cancer cells rather than a complex process involving the evolution and interaction of heterogeneous cancer cells and the tumour microenvironment.

Given this major deficiency, at first glance the second question of why such models are still relied on so heavily seems puzzling. But again the answer is simple: for much of the past 35 years, there have been no more suitable alternatives. And despite their flaws, xenograft models are relatively easy and inexpensive to use, and they have at least helped to prioritize compound testing.

Which bring us to the third question. Encouragingly, here it seems that one way in which the investment in basic cancer research is at last paying off is in the form of more realistic mouse models of cancer. Advances in our understanding of tumour biology, coupled with the ability to genetically engineer mice so that cancer develops in a way that more closely recapitulates the development of cancer in humans, have recently provided welldefined cancer models that could have predicted the observed failure of compounds in humans that were active in standard xenograft models.

Furthermore, as patent-related obstacles to the commercial use of genetically engineered mouse models have also considerably diminished recently, such models look set to become much more commonplace in the biopharma industry in the near future. If their predictive value is found to be a major improvement on that of xenografts as hoped, then one battle against cancer might be claimed as won. A similar resolve to investigate and tackle other key challenges in cancer therapy, such as early disease detection ${ }^{3}$, could be crucial in achieving the long-sought decisive victories in this war.

\footnotetext{
. Leaf, C. Fortune 9 Mar (2004).

. Kola, I. \& Landis, J. Nature Rev. Drug Discov. 3, 711-715 (2004).

3. <http://www.nature.com/nature/focus/cancerhorizons/index.html > (2006).
} 